\title{
Wer versucht, die Menschen zu bevormunden?
}

* Dr. med. Jean Martin, Mitglied der Redaktion, ist ehemaliger Kantonsarzt Waadt und Mitglied der nationalen Ethikkommission.

** Wobei einige dieser Kampagnen sich bereits als höchst wertvoll erwiesen haben, und es wäre in hohem Masse unverantwortlich gegenüber der Gesellschaft gewesen, sie nicht zu führen - von der Strassenverkehrserziehung über die HIV-/Aidsaufklärung bis hin zur Förderung von Schutzimpfungen (wie dies zuletzt durch die erneute Ausbreitung der Masern in der Schweiz Anfang 2008 veranschaulicht wurde).

1 Paccaud F. Prävention als Kampfsportart. Schweiz Ärztezeitung. 2008;89(23):1015.

2 Cassis I. Präventionsgesetz: eine einmalige Chance für die Schweiz! Schweiz Ärztezeitung. 2008; 89(26): 1165.

3 Weil B. Präventionsgesetz - ganzheitliche Vorlage mit hehren Zielen. Schweiz Ärztezeitung. 2008;89(34):1441.
Der Pianist Duke Ellington sagte einmal in einem philosophischen Moment: «Das Wort Freiheit wird auf unterschiedlichste Weisen gebraucht manchmal sogar im Sinne der Freiheit.» Wer sich mit Fragen des Gesundheitswesens beschäftigt, muss leider feststellen, dass diejenigen, die derzeit Kritik an den verstärkten Präventionsbemühungen üben, nicht im Sinne der Freiheit sprechen auch wenn Teile der Wirtschaft auf theatralische Weise versuchen, den Gesundheitsschutz als Freiheitsbeschneidung darzustellen. Ich erinnere nur an die Angriffe, denen sich Thomas Zeltner, Direktor des Bundesamts für Gesundheit [1], kürzlich ausgesetzt sah.

Manchen Menschen ist jedes Mittel recht, um ihre kurzfristigen ökonomischen Interessen zu schützen. Das ist nichts Neues, sondern Realpolitik ... In diesem Zusammenhang muss aber unterstrichen werden, dass die finanziellen Mittel, die für den Kampf für wirksamere Prävention eingesetzt werden, geradezu verschwindend gering sind im Vergleich zu den Summen, die die Unternehmen für ihre Öffentlichkeitsarbeit ausgeben. So ist es den Verfechtern des Gesundheitsschutzes unmöglich, sich genügend Gehör für die zentrale Frage zu verschaffen: «Wie und von wem wird die Freiheit des Einzelnen in der heutigen Gesellschaft stärker unangemessen beeinflusst, kanalisiert, bevormundet - von den bescheidenen Präventionskampagnen ${ }^{* *}$ oder von den tagtäglichen grossangelegten PR-Feldzügen für gesundheitsschädigende Produkte und Lebensgewohnheiten?»

In der Erklärung der Menschen- und Bürgerrechte, die in der Französischen Revolution verabschiedet wurde, heisst es: «Die Freiheit besteht darin, alles tun zu dürfen, was einem anderen nicht schadet.» Wie steht es nun in dieser Hinsicht mit den Angriffen auf unsere freie Willensbildung und unsere Gesundheit, die die angesprochenen Marketingmassnahmen objektiv darstellen? Um nur eins von vielen Beispielen zu nennen: Wir sehen in den USA, wie durch Ideologie die wirksame Prävention von jährlich mehr als 30000 Erschiessungstoden (in der Schweiz würde es 700 Tote verursachen; deutlich mehr als durch Verkehrsunfälle) verhindert wird - mittels des politischen Gewichts der Waffenlobby. Es ist zwar schwer vorstellbar, wie es der «National Rifle Association» gelingt, auch heutzutage in der Mehrheit der Bevölkerung die Überzeugung lebendig zu halten, dass Amerika seine Seele verlieren würde, wenn nicht jeder das Recht hätte, sein persönliches Waffenarsenal anzulegen wie damals die Cowboys mit ihren rauchenden Colts. Aber die NRA schafft es immer wieder.

Frappierend ist auch, wie gleich in mehreren Bereichen unserer Gesellschaft die Gewalt und eine ihrer Ausprägungsformen, die Pornographie, explosionsartig zunehmen: Gewalttätiges Verhalten - wozu auch Leichtsinn im Strassenverkehr zählt - und Sexbesessenheit haben zahlreiche explizite oder weniger explizite - wirtschaftliche Implikationen. Die dynamischen Unternehmer in diesen Branchen scheren sich wenig um die Notwendigkeit, beeinflussbare Mitbürger - insbesondere junge Menschen - zu schützen. Vom Gesundheits- und Sozialwesen hingegen erwarten sie selbstverständlich, dass diese die angerichteten Schäden wieder gutmachen.

Unter dem Strich ist also die Tatsache festzuhalten, dass die Freiheit des Einzelnen und sein Recht auf Selbstbestimmung ohne unangemessene äussere Beeinflussung und auf wirklich freie Entscheidung häufiger und schwerwiegender durch die PR der Wirtschaft eingeschränkt werden als durch die Ärzte und anderen Fachleute, die sich für die Gesundheit der Bevölkerung einsetzen. Genau diese Fachleute werden jedoch mit Pauschalkritik überzogen, während diejenigen, die lauthals wegen «Freiheitsbeschneidung» Alarm schlagen, versuchen, uns um jeden Preis (im wahrsten Sinne des Wortes, wenn man die investierten Summen betrachtet) vom Kauf ihrer Produkte zu überzeugen, selbst wenn diese höchstgefährlich sind. Wen kümmert das schon!

Fazit: Jede Auslegung des zentralen Schlagworts «Freiheit» muss beinhalten, dass anderen nicht geschadet wird. Doch so manches PRGetrommel ist von dem medizinisch-ethischen Grundsatz «Primum non nocere» denkbar weit entfernt. Mir ist klar, dass die genannten Wirtschaftsakteure nicht die Aufgabe haben zu heilen, doch es wäre schon viel gewonnen, wenn sie darauf achten würden, ihre Mitmenschen nicht zu schädigen. Dies sind nur einige der Gründe, aus denen es wünschenswert beziehungsweise unerlässlich ist, dass das Parlament noch in dieser Legislaturperiode eine gesetzliche Grundlage für wirksame Massnahmen verabschiedet $[2,3]$.

Jean Martin* 